\title{
Introduction of organised mammography screening in Tyrol: results following first year of complete rollout
}

\author{
Willi Oberaigner ${ }^{1,2,3^{*}}$, Martin Daniaux ${ }^{4}$, Sabine Geiger-Gritsch, Rudolf Knapp ${ }^{5}$, Uwe Siebert ${ }^{2,3,6,7}$ and \\ Wolfgang Buchberger ${ }^{2,8}$
}

\begin{abstract}
Background: In Tyrol, Austria, the existing system of spontaneous mammography screening was switched in 2007 to an organised program by smoothly changing the established framework. This process followed most EU recommendations for organised mammography screening with the following exceptions: women aged $40-49$ are part of the target population, screening is offered annually to the age group 40-59, breast ultrasound is available as an additional diagnostic tool, and double reading has not yet been implemented. After a pilot phase the program was rolled out to all of Tyrol in June 2008. The aim of this study was to analyse the performance of the organised screening system by comparing quality indices and recommended levels given in the well-established EU guidelines.

Methods: Working from the results of the pilot phase, we extended the organised mammography system to all counties in Tyrol. All women living in Tyrol and covered by compulsory social insurance were invited for a mammography, in the age group 40-59 annually and in the age group 60-69 biennially. Screening mammography was offered mainly by radiologists in private practice, with further assessment performed at hospitals. Using the screening database, all wellestablished performance indicators were analysed and compared with accepted/desired levels as per the EU guidelines.

Results: From June 2008 to May 2009, 120,440 women were invited. Per 1000 mammograms, 14 women were recalled for further assessment, nine underwent biopsy and four cancer cases were detected. Of invasive breast cancer cases, $32.3 \%$ and $68.4 \%$ were $\leq 10 \mathrm{~mm}$ and $\leq 15 \mathrm{~mm}$ in size, respectively, and $79.2 \%$ were node-negative. The positive predictive value for further assessment and for biopsy was $25.9 \%$ and 39.9\%, respectively. Estimated two-year participation rate was 57.0\%. In total, 14 interval cancer cases were detected during one year of follow-up; this is $18.4 \%$ of the background incidence rate.

Conclusions: In Tyrol, Austria, an organised mammography screening program was implemented in a smooth transition from an existing spontaneous screening system and was completely rolled out within a short time. The high level of performance already seen in the pilot phase was maintained after rollout, and improvements resulting from the pilot phase were affirmed after one year of complete rollout.
\end{abstract}

\section{Background}

Breast cancer is the leading cause of female cancer death in all industrialised countries (and also worldwide), and the breast is also the leading incident cancer site for females [1]. Therefore, screening methods for breast cancer are of greatest public health importance.

\footnotetext{
* Correspondence: willi.oberaigner@iet.at

'Department of Clinical Epidemiology of the Tyrolean State Hospitals Ltd.,

Cancer Registry of Tyrol, Innsbruck, Austria

Full list of author information is available at the end of the article
}

A recently published Cochrane Review, which assessed the effect of mammography screening for breast cancer on mortality and morbidity concluded that screening is likely to reduce breast cancer mortality [2].

In 2006, in Tyrol, Austria, the decision was made to change the existing spontaneous mammography screening system to an organised program while, on the one hand, making best possible use of the mammography screening network established over the previous fifteen years and, on the other hand, following most EU recommendations for 
organised mammography screening. After a pilot phase conducted in two central counties of Tyrol covering 40\% of the population from June 2007 to May 2008 [3], the organised system was completely rolled out to all of Tyrol in June 2008. It was possible to establish a country-wide mammography screening program in a very short time, which differs only in the following aspects from the EU guidelines [4]: women aged 40-49 are part of the target population, screening is offered annually in the age group 40-59, breast ultrasound is available as an additional diagnostic tool, and double reading has not yet been implemented.

To our knowledge, some European countries still have no organised mammography screening program or are in the process of planning to set up such a system $[5,6]$. Therefore, the Tyrolean experience can make an important contribution to deciding how to switch a health system with spontaneous mammography screening to an organised screening program that meets well-accepted quality guidelines.

It was the aim of this study to analyse the performance of the organised mammography screening system after complete rollout to all counties in Tyrol by measuring the quality indicators recommended by the EU guidelines [4] and to determine whether the high quality observed in the pilot phase could be affirmed after rollout.

\section{Methods}

\section{Study population, invitation}

The target population in the first year of complete rollout from June 2008 to May 2009 included all women aged 40 to 69 living in Tyrol and covered by compulsory social insurance, which is more than $97 \%$ of the population (personal communication). The main health insurance carrier sent out personal invitation letters to all the women in the target population in the month in which the women had their birthday: women aged 40-59 annually, and women aged 60-69 biennially. As women aged 60-69 and living in the two central counties of Tyrol where the pilot phase was conducted had already been invited in the pilot year, this group of women was not invited again in the first year of rollout (Figure 1). Mammography screening was offered by 22 screening units; thirteen of them were run by radiologists in private practice and nine by hospital outpatient departments. The mammogram was read by only one radiologist; ultrasound (US) was offered to women at the radiologist's discretion. Assessment was offered by nine hospital radiology units in the study area and included clinical inspection, mammography, US, magnetic resonance imaging (MRI) and biopsy as needed. Women were recalled for assessment either directly by the screening unit or by the general practitioner. The one large assessment unit at Innsbruck Medical University Hospital works closely with a breast cancer centre that was EUSOMA-certified in March 2010 [7]. All radiologists participating in the program underwent training and received ÖRG (Austrian Radiology Association) certification. In the median, private radiologists and hospital units performed 3234 and 1639 mammograms per year, respectively. The mammography screening system has been described in more detail elsewhere [3].

\section{Data collection}

All mammography units registered basic information in a database. Screening and assessment information was transferred to a central database after pseudonymising the woman's social insurance number [3]. In addition, data on tumour characteristics were collected by the Cancer Registry of Tyrol.

\section{Statistical analysis}

The screening and assessment data were realised as STATA datasets. Linkage between screening data, assessment data and Cancer Registry data is based on the pseudonym number. We reported numbers and proportions as defined in the EU guidelines [4]. For some indices, population-based rates were computed using the official population data supplied by Statistics Austria. No statistical testing was applied. All reporting was done with STATA Version 11 [8].

Performance indicators were reported from all screens in women aged 40-69 between June 2008 and May 2009.

Participation rate was calculated following a cohort approach: we counted every woman only once in the observation period, which was either one year or two years. Due to the fact that nearly half of women aged 40 to

\begin{tabular}{|c|c|c|c|}
\hline \multicolumn{2}{|r|}{ Pilot Phase } & $1^{\text {st }}$ Year Rollou & $2^{\text {nd }}$ Year Rollout \\
\hline \multicolumn{4}{|c|}{ June 2007} \\
\hline Age 40-69 & Central counties & Tyrol & Tyrol \\
\hline Age $50-59$ & Central counties & Tyrol & Tyrol \\
\hline Age 60-69 & Central counties & $\begin{array}{l}\text { Tyrol except } \\
\text { central counties }\end{array}$ & Central counties \\
\hline
\end{tabular}


59, who attend screening regularly, do not return for screening in the first year although they are invited annually, we computed for that age group also a two-year participation rate, meaning an observation period of two years.

Data on all mammography investigations performed in Tyrol are transferred to the screening database. A small portion (5.9\%) of the women refused consent for data transfer to the screening database and we therefore receive only an empty dataset. Of all other mammography data, $76 \%$ belong to the screening population. By assuming this same proportion of $76 \%$ for the empty dataset, we calculated a proportion of $4.5 \%$ to be added to the observed participation rate accounting for empty datasets describing real numbers of mammography screening investigations.

As spontaneous mammography screening was already introduced to Tyrol in the early 1990s, the underlying background incidence rate (BIR) was defined by years of diagnosis 1988-1990.

This study was conducted in conformity with the Helsinki Declaration [9]. The project was approved by the Ethics Committee of Innsbruck Medical University.

\section{Results}

From June 2008 to May 2009 120,440 women in the target population were invited; this excluded women aged 60-69 and living in the two central counties where the pilot phase was conducted, who fell in the biennial screening interval for that age group and were thus not invited again in this first year of complete rollout (Figure 1). The observed one- and two-year participation rates were $31.6 \%$ and $52.5 \%$, respectively (Table 1 ). Participation was higher in younger women. For example, the two-year observed participation rate was $55.1 \%$ in women aged $40-49$ versus $50.3 \%$ in women aged 50-69.

Performance indicators were analysed for all screens performed in the first year of rollout, namely 42,834 screens. Of the women $75.5 \%$ underwent additional US (80.9\% in women aged 40-49). Breast density (ACR 3/4) was the reason for additional US in $52.7 \%$ and $39.6 \%$ of women aged 40-49 and 50-69, respectively (Table 2).

Per 1000 screens, 14 women were recalled for further assessment. Screening result was unknown for a total of

Table 1 Invitation system: Number of women invited and participation rates

\begin{tabular}{llll}
\hline & $\mathbf{4 0 - 4 9}$ & $\mathbf{5 0 - 6 9}$ & Total (40-69) \\
\hline Women invited & 56,888 & 63,552 & 120,440 \\
Observed one-year participation rate & $32.6 \%$ & $30.6 \%$ & $31.6 \%$ \\
Estimated one-year participation rate & $37.1 \%$ & $35.1 \%$ & $36.1 \%$ \\
Observed two-year participation rate & $55.1 \%$ & $50.3 \%$ & $52.5 \%$ \\
Estimated two-year participation rate & $59.6 \%$ & $54.8 \%$ & $57.0 \%$ \\
\hline
\end{tabular}

98 cases $(0.2 \%$ of screens). Per 1000 screens, nine underwent biopsy. Of all biopsies, 86\% were core biopsies and $3 \%$ open biopsies (13 cases). We observed 3.6 screendetected cancers per 1000 screens or a total of 153 breast cancer cases, of which $9.2 \%$ were diagnosed as in situ cancers. The positive predictive value (PPV) was $25.9 \%$ for further assessment, 39.9\% for total biopsy and $45.8 \%$ for core biopsy. PPV was lower in age group 4049 (18.7\%, 31.3\% and 34.9\% for further assessment, total biopsy and core biopsy, respectively). Performance parameters are summarised in Table 3.

Of 139 invasive cancers diagnosed in screening, four changed to "in situ cancer" after final diagnosis. Two invasive cancer cases did not undergo surgery because of metastatic disease. Finally, three invasive cancer cases underwent neoadjuvant therapy and it was not possible to identify preoperative staging.

Of all invasive cancers detected and finally proven, $32.3 \%$ and $68.4 \%$ showed tumour size $\leq 10 \mathrm{~mm}$ and $\leq 15$ $\mathrm{mm}$, respectively. Lymph node involvement was observed in $20.8 \%$ of invasive cancer cases (Table 4 ).

For invasive cancers, $90.6 \%$ of further assessments were carried out within five working days after screening and $87.1 \%$ and $90.1 \%$ underwent surgery within ten days and 15 days after decision to operate, respectively. For all cases except invasive cancers, $73.7 \%$ underwent assessment within five working days and $17.1 \%$ after ten or more working days (Table 5).

We observed a total of 14 interval cancer cases within one year after screening in all of Tyrol, five in age group $40-49$, giving an interval cancer rate of $20.8 \%$ and $17.8 \%$ of the background incidence rate for age groups 40-49 and 50-69, respectively (Table 6). Table 7 shows the results for the most important quality indicators of the EU guidelines [4] restricted to age group 50-69. Given that the organised system was introduced after more than a decade of spontaneous mammography screening in Tyrol, as reference values we chose the accepted and desired ranges of EU quality indicators for subsequent rounds. Most of the indicators were within the EU range, except the participation rate $(54.8 \%$ vs. the limit of $75 \%)$, the proportion of II+ cancers $(33.3 \%$ vs. the limit of $25 \%$ ), the proportion of invasive cancers $(91.2 \%$, this is slightly above the limit of $90 \%)$ and the proportion of cases that underwent surgery within $\leq 15$ working days after decision to operate $(87.3 \%$, this is slightly below the limit of $90 \%$ ).

\section{Discussion}

We analysed performance after one year of rolling out an organised mammography screening program to all counties in Tyrol. The organised program was established in a smooth transition from an existing spontaneous mammography screening system, instead of setting up a completely new screening system, and was 
Table 2 Additional ultrasound at screening

\begin{tabular}{llll}
\hline & $\mathbf{4 0 - 4 9}$ & $\mathbf{5 0 - 6 9}$ & Total \\
\hline $\begin{array}{l}\text { Ultrasound following mammography screening } \\
\text { Reason for ultrasound: }\end{array}$ & $15,126(80.9 \%)$ & $17,196(71.2 \%)$ & $32,322(75.5 \%)$ \\
$\quad$ & & $6,806(39.6 \%)$ & $14,777(45.7 \%)$ \\
$\quad$ Breast density (ACR 3/4) & $7,971(52.7 \%)$ & $2,318(13.5 \%)$ & $4,119(12.7 \%)$ \\
$\quad$ Equivocal finding & $1,801(11.9 \%)$ & $8,072(46.9 \%)$ & $13,426(41.5 \%)$ \\
$\quad$ Other & $5,354(35.4 \%)$ & & \\
\hline
\end{tabular}

Table 3 Performance parameters

\begin{tabular}{|c|c|c|c|}
\hline & $40-49^{1)}$ & $50-69^{1)}$ & Total $^{1)}$ \\
\hline Recall for further assessment rate [per 1000 screens] and number of recalls ${ }^{2)}$ & $14.6(273)$ & $13.2(318)$ & $13.9(591)$ \\
\hline Intermediate screening test recommended in six months & $18.9(354)$ & $12.1(292)$ & $15.1(646)$ \\
\hline Screening result unknown ${ }^{3)}$ & $2.8(52)$ & $1.9(46)$ & $2.3(98)$ \\
\hline Biopsy rate [per 1000 screens] & $8.7(163)$ & $9.1(220)$ & $8.9(383)$ \\
\hline Cancer detection rate [per 1000 screens] & $2.7(51)$ & $4.2(102)$ & $3.6(153)$ \\
\hline Invasive & $2.5(46)$ & $3.9(93)$ & $3.3(139)$ \\
\hline In situ & $0.3(5)$ & $0.4(9)$ & $0.3(14)$ \\
\hline Proportion of in situ cases & $9.8 \%$ & $8.8 \%$ & $9.2 \%$ \\
\hline Ratio screening breast cancer detection rate vs. background incidence rate ${ }^{4)}$ & 2.1 & 2.0 & 2.0 \\
\hline PPV assessment & $18.7 \%(51 / 273)$ & $32.1 \%(102 / 318)$ & $25.9 \%(153 / 591)$ \\
\hline PPV biopsy & $31.3 \%(51 / 163)$ & $46.4 \%(102 / 220)$ & $39.9 \%(153 / 383)$ \\
\hline
\end{tabular}

1) Rates were rounded to one decimal; numbers in brackets are numbers of cases (i.e. recall rate, biopsy rate, cancer detection rate). PPV is rounded to one decimal; numbers in brackets are detailed numbers for computing PPV.

2) For one case assessment was recommended, but performed at an institution outside the screening system.

3) Cases with BI-RADS 0 without assessment were treated as unknown.

4) Background incidence rate defined by years of diagnosis 1988-1992.

previously tested in a pilot phase comprising $40 \%$ of the target population [3]. Although not all EU recommendations were followed, most quality indicators are in the range of accepted/desired levels given by the EU guidelines [4]. The only parameter that clearly missed the EU guidelines was the participation rate: the two-year participation rate was $57 \%$ as compared to the $75 \%$ recommended by the EU guidelines. In our opinion, a cumulative participation rate of $57 \%$ after two years of observation looks successful when compared to neighbouring countries [10-12]. Nevertheless, it is not the goal we aimed for.

Table 4 Characteristics of invasive cancer cases

\begin{tabular}{|c|c|c|c|}
\hline & $40-49$ & $50-69$ & Total \\
\hline Tumour size $(\mathrm{mm}): \mathrm{N}=133$ & $13 ; 4-25$ & $12 ; 1-35$ & $13 ; 1-35$ \\
\hline \multicolumn{4}{|l|}{ Median; range } \\
\hline \multicolumn{4}{|l|}{ Tumour size (mm): } \\
\hline$<=10 \mathrm{~mm}$ & $14(32.6 \%)$ & $29(32.2 \%)$ & $43(32.3 \%)$ \\
\hline$<=15 \mathrm{~mm}$ & $28(65.1 \%)$ & $63(70.0 \%)$ & $91(68.4 \%)$ \\
\hline $11-20 \mathrm{~mm}^{1)}$ & $23(53.5 \%)$ & $42(46.7 \%)$ & $65(48.9 \%)$ \\
\hline$>20 \mathrm{~mm}$ & $6(14.0 \%)$ & 19 (21.1\%) & $25(18.8 \%)$ \\
\hline Lymph node involvement & $8(18.6 \%)$ & $19(21.8 \%)^{1)}$ & $27(20.8 \%)$ \\
\hline \multicolumn{4}{|l|}{ Staging according to UICC } \\
\hline 1 & $30(69.8 \%)$ & $55(61.8 \%)$ & $85(64.4 \%)$ \\
\hline$\|$ & $13(30.2 \%)$ & 31 (34.8\%) & $44(33.3 \%)$ \\
\hline III & & 3 (3.4\%) & 3 (2.3\%) \\
\hline
\end{tabular}

Notes: Of 139 invasive cancer cases, four cases were finally "in situ"; two invasive cancer cases did not undergo surgery because of metastatic status. Three cases were without lymph node status because of neoadjuvant therapy and because we could not identify pretherapeutic TNM stage. 
Table 5 Waiting times

\begin{tabular}{|c|c|c|c|}
\hline \multicolumn{4}{|c|}{ Invasive cancers } \\
\hline & $40-49$ & $50-69$ & Total \\
\hline \multicolumn{4}{|c|}{ Screening to assessment } \\
\hline$\leq 5 \mathrm{wd}$ & $40(87.0 \%)$ & $86(92.5 \%)$ & $126(90.6 \%)$ \\
\hline $6-10 w d$ & $3(6.5 \%)$ & $3(3.2 \%)$ & $6(4.3 \%)$ \\
\hline$>10 \mathrm{wd}$ & $3(6.5 \%)$ & $4(4.3 \%)$ & $7(5.0 \%)$ \\
\hline \multicolumn{4}{|c|}{ Decision to operate to date of therapy } \\
\hline$\leq 10 \mathrm{wd}$ & $42(93.3 \%)$ & $73(83.9 \%)$ & $115(87.1 \%)$ \\
\hline $11-15$ wd & $1(2.2 \%)$ & $3(3.4 \%)$ & $4(3.0 \%)$ \\
\hline $16-30 \mathrm{wd}$ & $1(2.2 \%)$ & $4(4.6 \%)$ & $5(3.8 \%)$ \\
\hline$>30 \mathrm{wd}$ & $1(2.2 \%)$ & $7(8.0 \%)$ & $8(6.1 \%)$ \\
\hline \multicolumn{4}{|c|}{ All screens except those ending in invasive cancers } \\
\hline & $40-49$ & $50-69$ & Total \\
\hline \multicolumn{4}{|c|}{ Screening to assessment } \\
\hline$\leq 5 \mathrm{wd}$ & $162(72.3 \%)$ & 166 (75.1\%) & $328(73.7 \%)$ \\
\hline 6-10 wd & $17(7.6 \%)$ & $24(10.9 \%)$ & 41 (9.2\%) \\
\hline$>10 \mathrm{wd}$ & 45 (20.1\%) & 31 (14.0\%) & 76 (17.1\%) \\
\hline
\end{tabular}

wd: working days

The strengths of the Tyrolean breast cancer screening program are its implementation and performance: we were able to set up an organised population-based screening program within a short time with minimal additional resources that shows good performance. In summary, the recall for further assessment rate and the biopsy rate are fairly low, PPV was good as compared to other programs, only few open biopsies were performed, and despite the lack of double reading the interval cancer rate of $20 \%$ of the underlying BIR is rather good as compared to other programs [10,13-15].

However, this study has several weaknesses. First, up to now we have not implemented double reading as recommended in the EU guidelines. Interestingly, performance parameters and especially interval cancer rate showed that also without double reading an acceptable quality level was achieved. One reason could be the extensive use of additional US, about three of four women underwent additional US. The real benefit of US in a population-based mammography screening program is currently under discussion and has to be further evaluated $[16,17]$. Calculation of the interval cancer rate is reliant on the completeness of the Cancer Registry of Tyrol, which covers the target population. Completeness of incidence data in general has been shown to be very good $[3,18]$. In order to be able to analyse interval cancer rates for the screening program the timeliness of registration of breast cancer was improved, and linkage between cancer registry data and screening data is based on pseudonymising the social insurance number, which is read electronically. In the meantime, we have also assessed interval cancer in the time window 12 to 23 months for the pilot phase of the Tyrol program, see [3], and found five interval cancer cases in age group 40-49 (55\% of BIR) and seven interval cancer cases in age group 50-69 (33\% of BIR), data not shown.

Second, the average number of screens read by a radiologist in Tyrol per year (about 3200) does not meet the EU recommendation of 5000. A recent publication [19] showed that annual numbers below 5000 can still provide good sensitivity and acceptable false-positive rates.

Third, we used BI-RADS categories instead of a single yes/no rule for recall for further assessment. Some radiologists still use BI-RADS 0 (meaning unclear result) in a small number of cases ( $0.2 \%$ of all screens), and 15 per 1000 screens were invited to an intermediate screening test six months following a BI-RADS 3 screening result. Due to this inconsistency, the current program includes the following modifications: BI-RADS 0 is no longer allowed and BI-RADS 3 is strictly associated with recall for further assessment.

Many countries have run a mammography screening program for decades or for a shorter time. On the other hand, there are still some countries with no organised

Table 6 Interval cancer rate within first year

\begin{tabular}{|c|c|c|c|}
\hline & $40-49$ & $50-69$ & Total \\
\hline Interval cancer rate per 100,000 screens (number of cases in brackets) & $26.7(5)$ & $37.3(9)$ & $32.7(14)$ \\
\hline Proportion of background incidence rate ${ }^{1)}$ (in percent) & $20.8 \%$ & $17.8 \%$ & $18.4 \%$ \\
\hline
\end{tabular}

1) based on years of diagnosis 1988-1990 
Table 7 EU Guidelines, quality indicators (with accepted and desired levels)

\begin{tabular}{|c|c|c|c|}
\hline & Tyrol 50-69 & EU-accepted ${ }^{11}$ & EU-desired $^{1)}$ \\
\hline Participation rate (after two years of observation) & $54.8 \%$ & $>70$ & $>75$ \\
\hline Recall for further assessment rate ${ }^{1)}$ & $1.3(318)$ & $<5 \%$ & $<3 \%$ \\
\hline Breast cancer detection rate & $2.0 * \mathrm{BIR}$ & $1.5^{*} \mathrm{BIR}$ & $>1.5^{*} \mathrm{BIR}$ \\
\hline Interval cancer rate/Background incidence rate (BIR) 0-11 months & $18 \%(9)$ & $30 \%$ & $<30 \%$ \\
\hline Proportion of screen-detected cancers that were invasive & $91.2 \%(93 / 102)$ & $90 \%$ & $80 \%-90 \%$ \\
\hline Proportion of screen-detected cancers that were stage $\|+$ & $33.3 \%(34 / 102)^{2)}$ & 25 & $<25 \%$ \\
\hline Node-negative cancer/Total invasive cancers screen-detected & $78.2 \%$ & $75 \%$ & $>75 \%$ \\
\hline Invasive cancers $\leq 10 \mathrm{~mm} /$ Total invasive cancers & $32.2 \%$ & $\geq 25 \%$ & $\geq 30 \%$ \\
\hline Proportion of invasive cancers that were $\leq 15 \mathrm{~mm}$ in size & $70.0 \%$ & $50 \%$ & $>50 \%$ \\
\hline Time between results of screening and assessment $<=5 \mathrm{wd}^{3)}$ & $92.5 \%$ & $90 \%$ & $>90 \%$ \\
\hline Time between decision to operate and surgery $<=15 \mathrm{wd}^{4)}$ & $87.3 \%$ & $90 \%$ & $>90 \%$ \\
\hline
\end{tabular}

1) We took reference values for subsequent rounds, because we had a decade of spontaneous screening before beginning the organised program.

2) One of 150 cases without UICC staging (no staging because of neoadjuvant therapy).

3) We show time between screening and performed assessment, not offered assessment.

4) We show time between decision to operate and surgery performed (not between decision to operate and date offered for surgery).

breast cancer screening program. For those countries thinking of or already in the process of introducing a mammography screening program, our manner of introducing an organised program can serve as one how-to example. In our opinion, the greatest difference between our approach and other approaches, especially compared to Germany, is the smooth transition made from an existing spontaneous program to an organised population-based screening. We made use of the network of screening and assessment units that had already been set up during spontaneous screening and added an invitation system covering the entire population of Tyrol, a screening database that allows quality indices to be monitored and a well-defined training program for both screening and assessment units. With this strategy we were able to meet most EU quality indices within a very short time.

\section{Conclusions}

In Tyrol, Austria, an organised mammography screening system realised in a smooth transition from an existing spontaneous screening system was rolled out in a short time. The high level of performance already observed in the pilot phase has not changed after the first year of complete rollout. Improvements suggested during the pilot phase were affirmed after rollout: it will be necessary to concentrate on efforts to improve the participation rate, introduce double reading, change the rule for BI-RADS 3 , and reduce the number of additional ultrasound exams.

\section{List Of Abbreviations}

US: ultrasound, ÖRG: Austrian Radiology Association, ACR: American College of Radiology, MRI: magnetic resonance imaging, BIR: background incidence rate, PPV: posivite predictive value.

\section{Acknowledgements}

We thank Patricia Gscheidlinger for secretarial support.

Mammography Screening Project Tyrol Working Group:
Radiologists: B. Bischof, Innsbruck; J. Bliem, Wörgl; M. Daniaux, Innsbruck; A. Dessl, Zams; G. Egender, Hall/Tirol; W. Hönlinger, Innsbruck; W. Kastlunger, Schwaz; R. Knapp, Kufstein; A. Lederer, Lienz; C. Lottersberger, Schwaz; D. Lungenschmid, Innsbruck; A. Oberhauser, Innsbruck; E. Partl, Kitzbühel; T. Penz, Innsbruck; C. Rapf, Lienz; E. Schmaranzer, St. Johann; G. Schön, Telfs; R. Schöpf, Landeck; F. Weber, Hall in Tirol; K. Wicke, Innsbruck; C. Wolf, Reutte Project Team: W. Buchberger, M. Daniaux, C. Greil-Thum, R. Knapp, G. Nestler, W. Oberaigner, B. Schreiner, K. Wicke

This work was supported by the ONCOTYROL Center for Personal Cancer Medicine. ONCOTYROL is a COMET Center funded by the Austrian Federal Ministry of Transport, Innovation and Technology (BMVIT) and the Austrian Federal Ministry of Economics, Family and Youth (BMWFJ), the Tyrolean Future Foundation (TZS) and the State of Styria represented by the Styrian Business Promotion Agency (SFG) and supported by UMIT - University for Health Sciences, Medical Informatics and Technology, Innsbruck Medical University, Tyrolean Health Insurance Fund and Tyrolean Health Company.

\section{Author details}

${ }^{1}$ Department of Clinical Epidemiology of the Tyrolean State Hospitals Ltd., Cancer Registry of Tyrol, Innsbruck, Austria. ${ }^{2}$ Institute of Public Health, Medical Decision Making and Health Technology Assessment, Department of Public Health and Health Technology Assessment, UMIT - University for Health Sciences, Medical Informatics and Technology, Hall i.T., Austria. ${ }^{3}$ ONCOTYROL - Center for Personalized Cancer Medicine, Innsbruck, Austria. ${ }^{4}$ Innsbruck Medical University, Department of Radiology, Innsbruck, Austria. ${ }^{5}$ Kufstein County Hospital, Department of Radiology, Kufstein, Austria. ${ }^{6}$ Center for Health Decision Science, Department of Health Policy and Management, Harvard School of Public Health, Boston, MA, USA. ${ }^{7}$ Institute for Technology Assessment and Department of Radiology, Massachusetts General Hospital, Harvard Medical School, Boston, MA, USA. ${ }^{8}$ Tyrolean State Hospitals Ltd., Medical Department, Innsbruck, Austria.

\section{Authors' contributions}

WO and WB designed the study. WO performed the analysis and wrote the paper. SGG contributed to writing the paper and to critically reviewing the draft. WB, MD und RK contributed to the Discussion section, especially from the radiology point of view. US contributed to writing the paper. All authors reviewed and agreed to the final version of the manuscript.

\section{Competing interests}

The authors declare that they have no competing interests.

Received: 20 May 2011 Accepted: 30 August 2011

Published: 30 August 2011

\section{References}

1. Boyle P, Levin B, Eds: World Cancer Report 2008 Lyon: IARC Press; 2008. 
2. Gotzsche PC, Nielsen M: Screening for breast cancer with mammography. Cochrane Database Syst Rev 2011, CD001877.

3. Oberaigner W, Buchberger W, Frede T, Daniaux M, Knapp R, Marth C, Siebert $\mathrm{U}$ : Introduction of organised mammography screening in tyrol: results of a one-year pilot phase. BMC Public Health 2011, 11:91-99.

4. Perry N, Broeders M, de Wolf C, (Eds.): European guidelines for quality assurance in breast cancer screening and diagnosis. Luxembourg: Office for Official Publications of the European Communities; 2006.

5. Karsa L, Anttila A, Ronco G, Ponti A, Malila N, Arbyn M: Cancer Screening in the European Union. Report on the implementation of the Council Recommendation on cancer screening. First Report Brussels: European Commission; 2008.

6. Dowling EC, Klabunde C, Patnick J, Ballard-Barbash R: Breast and cervical cancer screening programme implementation in 16 countries. J Med Screen 2010, 17:139-146.

7. Eusoma-Breast Unit-Certification Process. [http://www.eusoma.org/Engx/ BreastUnits/AccreditationProcess.aspx?cont=ap_accredited], accessed 09.05.2011.

8. Stata Statistical Software: Release 11 College Station, Tx, StataCorp LP; 2009.

9. World Medical Association Declaration of Helsinki: ethical principles for medical research involvin human subjects. [http://www.wma.net/en/ 30publications/10policies/b3/index.html], (acessed 7 November 2009).

10. Kooperationsgemeinschaft Mammographie, (Ed.): Evaluationsbericht 20052007. Ergebnisse des Mammographie-Screening-Programms in Deutschland. Köln; 2009.

11. Bulliard JL, Ducros C, Jemelin C, Arzel B, Fioretta G, Levi F: Effectiveness of organised versus opportunistic mammography screening. Ann Oncol 2009, 20:1199-1202.

12. Giordano L, Giorgi D, Ventura L, Stefanini V, Senore C, Castagno R, Paci E, Segnan N: Time trends of process and impact indicators in Italian breast screening programmes: 1998-2008. Epidemiol Prev 2010, 34:27-34.

13. Hofvind S, Vacek PM, Skelly J, Weaver DL, Geller BM: Comparing screening mammography for early breast cancer detection in Vermont and Norway. J Natl Cancer Inst 2008, 100:1082-1091.

14. Blanks RG, Moss SM, McGahan CE, Quinn MJ, Babb PJ: Effect of NHS breast screening programme on mortality from breast cancer in England and Wales, 1990-8: comparison of observed with predicted mortality. BMJ 2000, 321:665-669.

15. Smith-Bindman R, Chu PW, Miglioretti DL, Sickles EA, Blanks R, BallardBarbash R, Bobo JK, Lee NC, Wallis MG, Patnick J, Kerlikowske K: Comparison of screening mammography in the United States and the United kingdom. JAMA 2003, 290:2129-2137.

16. Corsetti V, Houssami N, Ghirardi M, Ferrari A, Speziani M, Bellarosa S, Remida G, Gasparotti C, Galligioni E, Ciatto S: Evidence of the effect of adjunct ultrasound screening in women with mammography-negative dense breasts: Interval breast cancers at 1year follow-up. Eur J Cancer 2011, 47:1021-1026.

17. Husby JA, Espeland A, Kalyanpur A, Brocker C, Haldorsen IS: Double reading of radiological examinations in Norway. Acta Radiol 2011.

18. Oberaigner W, Siebert U: Are survival rates for Tyrol published in the Eurocare studies biased? Acta Oncol 2009, 1-8.

19. Buist DS, Anderson ML, Haneuse SJ, Sickles EA, Smith RA, Carney PA Taplin SH, Rosenberg RD, Geller BM, Onega TL, et al: Influence of annual interpretive volume on screening mammography performance in the United States. Radiology 2011, 259:72-84.

\section{Pre-publication history}

The pre-publication history for this paper can be accessed here: http://www.biomedcentral.com/1471-2458/11/673/prepub

doi:10.1186/1471-2458-11-673

Cite this article as: Oberaigner et al:: Introduction of organised mammography screening in Tyrol: results following first year of complete rollout. BMC Public Health 2011 11:673.

\section{Submit your next manuscript to BioMed Central and take full advantage of:}

- Convenient online submission

- Thorough peer review

- No space constraints or color figure charges

- Immediate publication on acceptance

- Inclusion in PubMed, CAS, Scopus and Google Scholar

- Research which is freely available for redistribution

Submit your manuscript at www.biomedcentral.com/submit
Ciomed Central 